\title{
Hybrid dual laser processing for improved quality of inclined up-facing surfaces in laser powder bed fusion of metals
}

\author{
Jitka Metelkova $^{\mathrm{a}, \mathrm{b}}$, Lars Vanmunster ${ }^{\mathrm{a}, \mathrm{b}}$, Han Haitjema ${ }^{\mathrm{a}}$, Daniel Ordnung ${ }^{\mathrm{a}, \mathrm{b}}$, Jean-Pierre Kruth ${ }^{\mathrm{a}, \mathrm{b}}$, Brecht Van \\ Hooreweder ${ }^{\mathrm{a}, \mathrm{b}, *}$ \\ ${ }^{a}$ Department of Mechanical Engineering, KU Leuven, Celestijnenlaan 300, 3001 Leuven, Belgium \\ ${ }^{b}$ Member of Flanders Make
}

\begin{abstract}
Additive manufacturing techniques such as laser powder bed fusion (LPBF) enable production of components with highly complex geometries. However, their as-built surface quality and geometrical precision remain insufficient for high-end applications, requiring further post-processing. Unlike horizontal surfaces, inclined up-facing surfaces cannot be improved by in-situ remelting, as they remain covered with powder. In this research a dual laser setup replaces the traditional single-laser layout. Surface enhancement is achieved in two steps: a nanosecond pulsed laser is used to remove powder from selected areas via laser-induced shock waves (LISW), so that these surfaces can be remelted with a continuous wave laser. This work presents an investigation of in-situ remelting (laser polishing) of inclined surfaces in their as-built orientation, after powder removal by brushing or using LISW. Particular focus is given on the effect of process parameters (laser power, scanning speed, number of scans) on the surface and subsurface quality and dimensional accuracy. As a result, this in-situ processing leads to a comparable surface texture on horizontal and inclined surfaces, without introducing any major subsurface defects.
\end{abstract}

Keywords: additive manufacturing, laser powder bed fusion, surface roughness, staircase effect, in-situ remelting, maraging steel 300

\section{Introduction}

Laser powder bed fusion (LPBF) is an additive manufacturing technique allowing production of lightweight components with complex geometries and high performance (Sing and Yeong 2020). As discussed by Schmidt et al. (2017), such components find applications in high-end sectors, such as aerospace, mould-making or medical industry. However, Leach et al. (2019) concluded that the benefits of this technique are often compromised by insufficient surface quality and geometrical precision, which makes them unable to meet the final application requirements. In most cases post-processing became an inevitable part of the manufacturing chain, increasing both production time and cost (Lee et al. 2021).

As defined in ISO 17296-2 (2015), during the LPBF process the component is built in a layer-by-layer manner through deposition of fine layers of metal powder and their subsequent melting with a laser. These steps are repeated until the whole part is built. Yasa et al. (2011) showed that due to the nature of the LPBF process, the quality of horizontal up-facing (upskin) surfaces can be improved by in-situ remelting. On the other hand, in view of the powder deposited during previous layers, remelting cannot be applied to inclined up-facing surfaces. The inclined surfaces are covered with powder, a direct interaction with the laser beam is thus impossible. Yet, several studies (Boschetto et al. 2017; Strano et al. 2013) indicate that inclined surfaces have rougher surface texture than horizontal up-facing surfaces. Multiple phenomena contribute to this outcome, namely the staircase effect (stair-step effect)

\footnotetext{
${ }^{*}$ Corresponding author

Email address: brecht.vanhooreweder@kuleuven. be (Brecht Van Hooreweder)

URL: www . set . kuleuven. be/am/ (Brecht Van Hooreweder )
} 
related to the layer-wise manufacturing (Campbell et al. 2002), the edge effect referring to elevated edges at the border of a scanned area (Yasa and Kruth 2011) and powder particle attachment (Newton et al. 2020).

The surface quality of LPBF parts can be improved by various processes such as laser, chemical, abrasive or mechanical polishing (Boban and Ahmed 2021). In view of the industrial challenges, an increasing number of researchers study "hybrid manufacturing", a combination of multiple manufacturing techniques (Webster et al. 2021). However, only some of the mentioned techniques are suitable for an in situ integration in the LPBF process. Some authors reported hybrid setups combining LPBF and mechanical machining such as milling (Du et al. 2018; Wüst et al. 2020) or grinding (Colosimo et al. 2020). However, metal chips originating from the machining process might compromise the quality of powder deposition and its further reuse. Besides mechanical solutions, multiple authors (Bhaduri et al. 2017; Ramos-Grez and Bourell 2004; Gora et al. 2016; Ma et al. 2017; Yasa et al. 2011) demonstrated that a purely laser-based solution would be also beneficial for precision and surface quality improvement of LPBF parts. As reported by Yung et al. (2019) and Kumstel and Kirsch (2013), high surface roughness of LPBF parts is most often reduced by macropolishing with a continuous wave laser. However, it usually leads to the surface overmelting regime (Ramos-Grez and Bourell 2004) typical for its residual waviness and elevated edges. Laser polishing has been explored by many authors, such as a recent study by Kuisat et al. (2021), but remelting of inclined surfaces has been only seldom reported. Lamikiz et al. (2007) studied remelting of inclined parts made by Selective Laser Sintering (SLS) from steel infiltrated with bronze. Yasa and Kruth (2011) and similarly Ghorbani et al. (2020) and Yung et al. (2018) also presented solutions for inclined surface remelting by its discretization into height zones. However, all these findings work with powder-free inclined surfaces.

A recent study by Metelkova et al. (2020) explored an innovative approach using a dual-laser ("hybrid") setup for in-situ inclined surface enhancement. The authors employed a methodology involving both pulsed and continuous wave lasers. First the pulsed laser was used to generate laser-induced shock waves in the vicinity of the inclined surfaces in order to remove the powder obstructing direct access to the surface. Once the surface was powder-free, it could be remelted with the continuous wave laser, i.e. the same laser as used for building. Metelkova and Ordnung et al. (2021) also reported an in-depth study on the powder removal process, showing a successful surface cleaning of slopes up to $45^{\circ}$ and a height step of $4 \mathrm{~mm}$. However, the exact part geometry also affects the process efficiency. Although prior studies suggest a significant surface quality improvement, the detailed impact of this hybrid processing on the surface integrity has not yet been reported. This work investigates surface texture and subsurface regions of samples processed by both pulsed and continuous wave lasers.

The study consists in an investigation of the effect of various remelting parameters on surface and subsurface quality. In the first part of the study, the powder is removed by manual brushing, i.e. the as-built quality of the inclined surface is maintained. In the second part of the study, the effect of powder removal by manual brushing is compared to removal by laser-induced shock waves.

\section{Experimental setup and methodology}

The experiments took place in a modified LPBF machine, DMP ProX320 from 3D Systems. The machine was standardly equipped with a $500 \mathrm{~W}$ fiber laser with a central wavelength of $1070 \mathrm{~nm}$, focused on a spot with $d_{1 / e^{2}}=60 \mu \mathrm{m}$. In addition to this continuous wave $(\mathrm{CW})$ laser used for powder melting, a nanosecond pulsed wave (PW) laser has been implemented, with a maximal average power of $50 \mathrm{~W}$, laser spot size $d_{1 / e^{2}}=50 \mu \mathrm{m}$ and pulse duration range of 2-250 ns. As both lasers emit a comparable wavelength, the system can operate with a single galvano mirror scanner and f-theta lens optics. A flipping optic was integrated to allow switching between the two lasers. It is hence possible to remove material via laser ablation without interrupting the manufacturing process.

As illustrated in Fig. 1, the methodology used in this study consists in the following steps: (1) sample production by laser powder bed fusion, (2) powder removal by (2a) post-process manual brushing, or (2b) in-situ shock waves generated by a PW laser, so that the last step of (3) inclined surface remelting with a CW laser can take place. All the steps are performed under argon inert atmosphere.

\subsection{Samples production by $L P B F$}

The samples were built from maraging steel 300 with a nominal particle size distribution of $15-45 \mu \mathrm{m}$. A detailed study on the morphology of the powder used in this study was reported by Sinico et al. (2019). The building param- 
a)

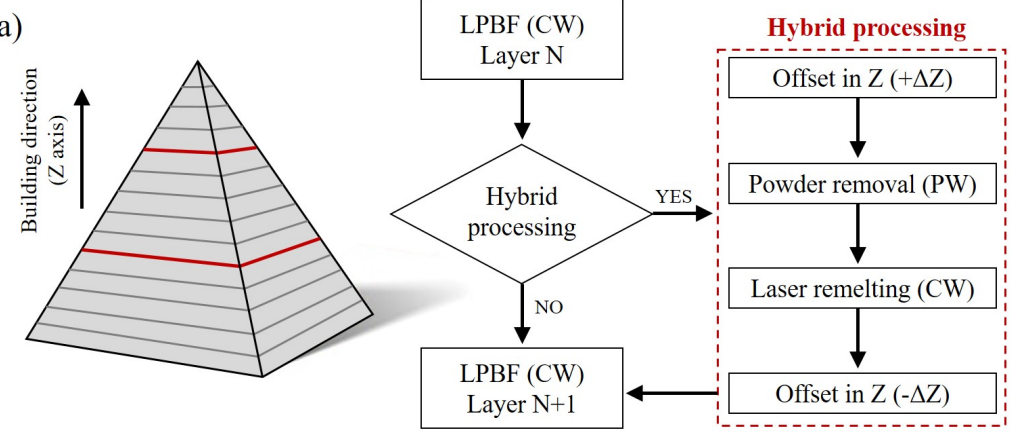

b)

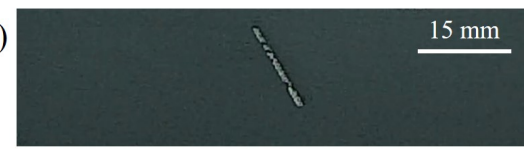

c)

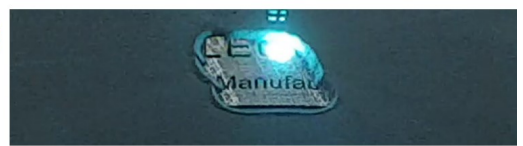

d)

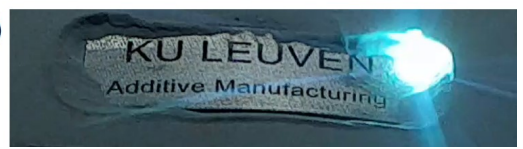

Figure 1: Illustration of the proposed methodology with (a) a flowchart with hybrid processing applied to selected layers marked in red, (b) demonstration part after building, (c,d) demonstration part during powder removal with PW laser using laser-induced shock waves (LISW).
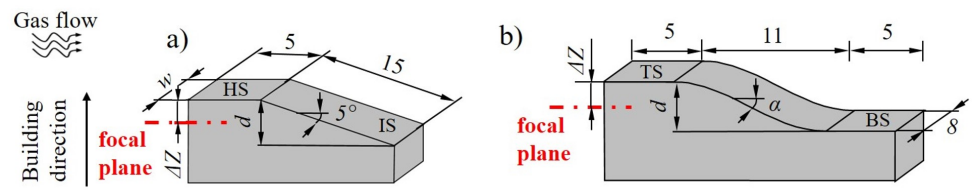

Figure 2: Sample geometry (in mm), (a) $5^{\circ}$ block (main investigated geometry), (b) sinusoidal sample, with surface inclination $\alpha$, sample depth $d$, focal plane offset $\Delta Z$, top horizontal surface TS and bottom horizontal surface BS.

eters given in Table 1 were optimized for a maximal part density $(98.9 \pm 0.1 \%)$, assessed following the Archimedes principle with a theoretical density of $8.1 \mathrm{~g} / \mathrm{cm}^{3}$. Contours scanning was not applied.

The main sample geometry investigated in the first part of the study is illustrated in Fig. 2a. It is a block with a $5 \mathrm{~mm}$ horizontal surface (HS) and $15 \mathrm{~mm}$ surface inclined by $\alpha=5^{\circ}$ (IS). The maximal depth of the treated surface $d=1.3 \mathrm{~mm}$. The default sample width $w$ is $8 \mathrm{~mm}$, unless otherwise stated in the text. The method was also applied to sinusoidal samples limited by two horizontal surfaces with a $4 \mathrm{~mm}$ height step $d$ (Fig. 2b). Between the top (TS) and bottom (BS) surface, the surface follows a sinusoidal curve, resulting in a continuous variation of the slope angle $\alpha$ between $0^{\circ}$ and $40^{\circ}$. The sample design as well as the build files were prepared using the 3DXpert software from 3D Systems.

\subsection{Selective powder removal and inclined surface remelting}

After the building process, inclined surfaces are completely covered by powder. In order to investigate the effect of surface remelting, a direct access to these surfaces is required and therefore the powder has to be removed. Two options of powder removal were investigated in this study: (1) by manual brushing (MB) or (2) by laser-induced shock waves (LISW).

Table 1: Used process parameters. Note: the scanning direction is defined with respect to the sample edge and gas flow direction. * refers to the process parameters selected for Section 3.3.

\begin{tabular}{lccc}
\hline & LPBF & Powder removal & Laser remelting \\
\hline Type of laser & $\mathrm{CW}$ & $\mathrm{PW}$ & $\mathrm{CW}$ \\
Average laser power $P / \mathrm{W}$ & 150 & 42 & $100-200\left(100^{*}\right)$ \\
Pulse duration $\tau / \mathrm{ns}$ & - & 30 & - \\
Pulse repetition rate $P R R / \mathrm{Hz}$ & - & 100 & - \\
Scanning speed $\nu / \mathrm{mm} / \mathrm{s}$ & 1100 & 500 & $600-1100\left(700^{*}\right)$ \\
Hatch spacing $h / \mu \mathrm{m}$ & 70 & 40 & 35 \\
Layer thickness $t / \mu \mathrm{m}$ & 30 & - & - \\
Focal plane offset $\Delta Z / \mathrm{mm}$ & 0 & $d / 2$ & $d / 2$ \\
Scanning direction $/^{\circ}$ & 45 & 0 & 45 \\
Inter layer rotation $/^{\circ}$ & 90 & 180 & 90 \\
Number of passes $N$ & - & 20 & $1-4\left(4^{*}\right)$ \\
\hline
\end{tabular}


MB was used to observe the sole effect of laser remelting on the surface texture and microstructure, without PW laser processing. After samples building and subsequent cooling down to room temperature, the machine was opened, the powder was manually brushed away. The machine was re-closed and the inert argon atmosphere was restored prior to remelting.

The approach using LISW is more suitable for industrial applications. It uses shock waves generated as a byproduct of nanosecond laser ablation. Fig. 1b-d shows its application on a demonstration part, being a concave $55 \mathrm{~mm}$ x $20 \mathrm{~mm}$ plate with a maximal depth of $2.5 \mathrm{~mm}$. The process of powder removal by LISW followed by surface remelting involves both a CW and a PW laser, therefore we refer to it as "hybrid" processing. In the present study it is applied after the last built layer, but as illustrated in Fig. 1a it could be also applied to intermediate layers marked in red. The procedure consists in moving the build cylinder upwards by $\Delta Z=d / 2$ and automatically switching to the PW laser mode in order to remove powder from selected areas using laser-induced shock waves (LISW). This step is implemented in order to place the focal plane in the middle height of the treated surface. The scanning vectors are oriented parallel to the surface gradient. Next, the system is automatically switched back to the CW laser for laser remelting. After this sequence, the build cylinder is moved back to its initial position and the building process can be resumed. The build cylinder offset was implemented in order to optimize the powder removal efficiency (Metelkova and Ordnung et al. 2021). For samples with a small depth $d$, this step might be omitted. The optimal process parameters for powder removal by LISW (Table 1) were selected based on previous research (Metelkova et al. 2020; Metelkova and Ordnung et al. 2021).

The remelting step consists in rescanning the part under $45^{\circ}$ with respect to the gas flow and sample edge, with a $90^{\circ}$ rotation between the remelting passes. The complete set of parameters is given in Table 1. Similarly to the powder removal step by LISW, the focal plane was set to the middle height of the remelted surface (Fig. 2).

\subsection{Characterization of the samples}

In order to provide a deeper insight into the surface topography of the samples, surface quality was evaluated based on 2.5D data. The height data was acquired using a Mitutoyo CS3200 tactile profilometer with a $60^{\circ}$ conical probe and $2 \mu \mathrm{m}$ tip radius. The $5^{\circ}$ blocks were assessed based on $2.5 \mathrm{D}$ data composed from 50 parallel profiles spaced by $60 \mu \mathrm{m}$. Due to a higher geometrical complexity, 100 profiles spaced by $30 \mu \mathrm{m}$ were used for $2.5 \mathrm{D}$ data composition of the sinusoidal samples. The $2.5 \mathrm{D}$ data was levelled and aligned with the evaluation axes. The surface texture was determined as the difference between the measured data and mean profile of the re-aligned data. As a numerical parameter representing the part quality, the $S_{a}$ parameter was calculated either from the full measured data or its part (e.g. to distinguish inclined and horizontal surfaces). $S_{a}$ is the arithmetical mean deviation of the filtered data, as defined in ISO 25178-2 (2012). The $S_{a}$ parameter was obtained after form removal followed by waviness removal by a Gaussian regression filter according to ISO 16610-31 (2016) with a long wavelength cut-off $\lambda_{c}=2.5 \mathrm{~mm}$.

The sample edge morphology was assessed following the method from Metelkova et al. (2019). The tactile probe made contact on the investigated surface ( $4 \mathrm{~mm}$ from the edge), acquired data in a direction perpendicular to the edge, until crossing the edge and landing on a gauge block positioned about $1 \mathrm{~mm}$ below the investigated surface. The reference block serves to prevent probe damage and to adjust profiles height so that a $2.5 \mathrm{D}$ data set can be created. In this study the edge was characterized based on 100 profiles $6 \mathrm{~mm}$ long with a $20 \mu \mathrm{m}$ spacing. The maximal edge height $h_{\max }$ as well as the edge length $L$ were assessed based on the mean edge profile as described by Metelkova et al. (2019). The sample corners were visually inspected using a digital microscope Keyence VHX-6000.

The impact of the hybrid processing on the subsurface quality was assessed based on metallographic crosssections. The microstructure was revealed using chemical etching with $10 \%$ nital solution during $80 \mathrm{~s}$. The melt pool depth $d_{M P}$ was assessed in three distinct locations based on a measurement of 20 consecutive melt pools. It was also correlated with the normalized enthalpy $\Delta H / h_{s}$, being a measure of heat input (King et al. 2014). It is defined in Eqs. (1-2) with the thermo-physical properties given in Table 2. Due to the LPBF machine setup with a fixed focal plane, scanning of inclined surfaces involves a continuous defocusing $\Delta f$. Defocusing refers to the vertical distance between the focal plane and the laser-matter interaction zone. As $5^{\circ}$ inclined surfaces involve a low degree of defocusing, $|\Delta f|<0.65 \mathrm{~mm}$, the calculations in Eq. (1) were simplified by considering the laser spot size $d_{1 / e^{2}}$ in focus.

$$
\frac{\Delta H}{h_{s}}=\frac{A \cdot P}{\pi \cdot h_{s} \cdot \sqrt{D \cdot v \cdot d_{1 / e^{2}}{ }^{3}}}
$$




$$
h_{s}=c_{p} \cdot \rho \cdot\left(T_{m}-T_{0}\right) \quad / \mathrm{J} / \mathrm{m}^{3}
$$

Moreover, the microhardness $H$ of selected samples was assessed at various depths on three distinct locations using a Vickers hardness tester Future Tech FV-700, with $500 \mathrm{~g}$ load and $10 \mathrm{~s}$ loading time.

Table 2: Thermo-physical properties of maraging steel used for calculation of Normalized Enthalpy (Ben-Artzy et al. 2021). * calculated properties.

\begin{tabular}{ll}
\hline Property & Value and units \\
\hline Absorptivity, $A$ & 0.35 \\
Density, $\rho$ & $8100 \mathrm{~kg} / \mathrm{m}^{3}$ \\
Specific heat capacity, $c_{p}$ & $335 \mathrm{~J} /(\mathrm{kg} \cdot \mathrm{K})$ \\
Initial temperature prior to melting, $T_{0}$ & $298.15 \mathrm{~K}$ \\
Melting point, $T_{m}$ & $1727 \mathrm{~K}$ \\
Thermal conductivity, $k$ & $19.6 \mathrm{~W} /(\mathrm{m} \cdot \mathrm{K})$ \\
Thermal diffusivity, $D^{*}$ & $7.22 \cdot 10^{-6} \mathrm{~m}^{2} / \mathrm{s}$ \\
Specific enthalpy at melting, $h_{s}^{*}$ & $3.88 \cdot 10^{9} \mathrm{~J} / \mathrm{m}^{3}$ \\
\hline
\end{tabular}

Selected samples were also heat treated using the methodology recommended by Elangeswaran et al. (2020). After a solutionizing treatment at $820^{\circ} \mathrm{C}$ for $1 \mathrm{~h}$, an age hardening treatment followed at $480^{\circ} \mathrm{C}$ for $3 \mathrm{~h}$. The treatment took place under argon with a heating rate of $10^{\circ} \mathrm{C} / \mathrm{min}$.

\section{Results and discussion}

\subsection{Laser remelting of $5^{\circ}$ inclined surfaces}

\subsubsection{Effect on the surface texture}

The core part of the study concerns remelting of the main sample geometry (blocks inclined by $\alpha=5^{\circ}$ ) performed after manual powder removal (MB).

In the first place, the effect of the number of remelting passes $N_{R}$ was investigated, using two different parameter sets $(P=150 \mathrm{~W}, v=1100 \mathrm{~mm} / \mathrm{s}$ and $P=100 \mathrm{~W}, v=700 \mathrm{~mm} / \mathrm{s})$. As shown in Fig. 3, after the first remelting pass the $S_{a}$ parameter rapidly decreases from $12.8 \mu \mathrm{m}$ in the as-built state to $8.2-8.9 \mu \mathrm{m}$, and then stabilizes at 6.6-7.1 $\mu \mathrm{m}$ after further remelting. However, the remelted surfaces present a visible waviness of $\lambda \approx 0.5 \mathrm{~mm}$ (Fig. 3b-d), comparable to the waviness of the as-built samples (Fig. 3a). As discussed by Ramos-Grez and Bourell (2004), this waviness is probably due to the high temperature gradients as the remelting conditions lead to the "surface overmelting" (SOM) regime characterized by relatively deep melt pools.

Fig. 3e-h presents autocorrelation plots of the surface topography. Autocorrelation reveals periodic features by a stripe-like texture. Fig. 3 shows that depending on the used parameters the stairs are fully removed after $N_{R}=2-4$, as no significant stripes are visible on the autocorrelation plots (Fig. 3g,h). In order to ensure a complete stair removal, $N_{R}=4$ will be used in the following sections.

The effect of other remelting process parameters on the part's surface quality was investigated as well, namely laser power $P$ and scanning speed $v$. Fig. 4 gives $S_{a}$ as a function of the process parameters of the remelting step. In this figure the dominant effect of $v$ on the surface quality enhancement is apparent. In accordance with the observations made by Marimuthu et al. (2015), $S_{a}$ improves with a lower $v$ but does not seem to be significantly impacted by the variation of $P$. Furthermore, due to the central position of the focal plane, the slight defocusing $(|\Delta f|<0.65 \mathrm{~mm})$ does not seem to influence significantly the surface texture of the remelted samples.

\subsubsection{Effect on the geometrical accuracy}

Concerning the geometrical precision, a previous study by Metelkova et al. (2019) showed a significant contribution of the remelting process to the edge effect. An analysis of the elevated edges was performed in the present study as well, following the same methodology as Metelkova et al. (2019). The measurements were performed on the HS surface, in the direction parallel to the longer sample dimension. The results presented in Fig. 5 show that the edge size (maximal edge height $h_{\max }$ and edge length $L$ ) increases with increasing normalized enthalpy: ranging from $h_{\max }=30.8 \mu \mathrm{m} \pm 10.8 \mu \mathrm{m}$ and $L=300 \mu \mathrm{m}$ (Fig. 5a) up to $h_{\max }=50.8 \mu \mathrm{m} \pm 3.3 \mu \mathrm{m}$ and $L=910 \mu \mathrm{m}$ (Fig. 5d). Increasing the normalized enthalpy leads to an increased melt pool temperature and size. As one of the main contributors to the elevated edges is evaporation of the material in the central zone, a more dominant edge effect for high enthalpy is to 

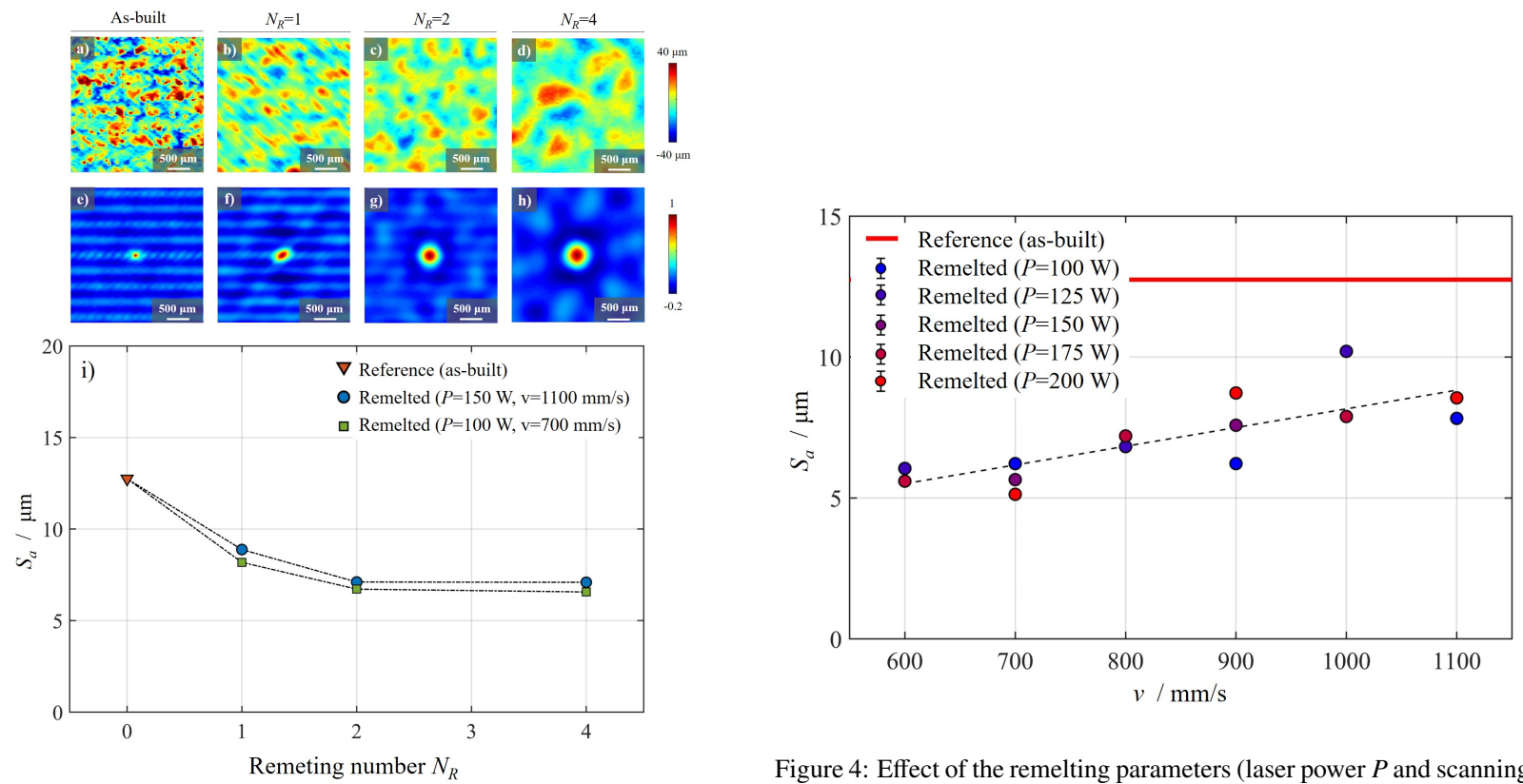

Figure 4: Effect of the remelting parameters (laser power $P$ and scanning speed $v)$ on the surface quality of $5^{\circ}$ blocks after MB $\left(N_{R}=4, h=35 \mu \mathrm{m}\right)$.

Figure 3: Effect of the number of remelting passes $N_{R}$ on the surface quality of $5^{\circ}$ blocks after $\mathrm{MB}$, with $2.5 \mathrm{D}$ height maps (a) in the as-built state, after remelting $(P=100 \mathrm{~W}, v=700 \mathrm{~mm} / \mathrm{s}$, $h=35 \mu \mathrm{m}$ ) with (b) $N_{R}=1$, (c) $N_{R}=2$, (d) $N_{R}=4$, (e-h) corresponding normalized autocorrelation plots, (i) effect on the $S_{a}$ parameter.

a)

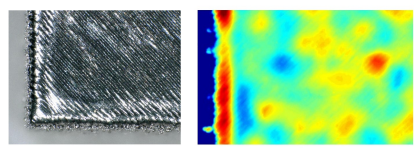

b)

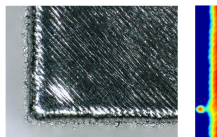

c)

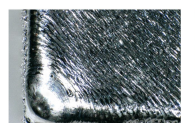

d)

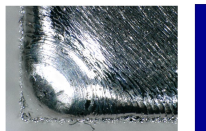

$1 \mathrm{~mm}$
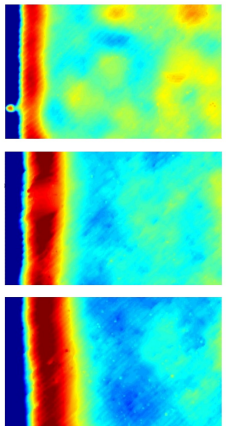

$1 \mathrm{~mm}$
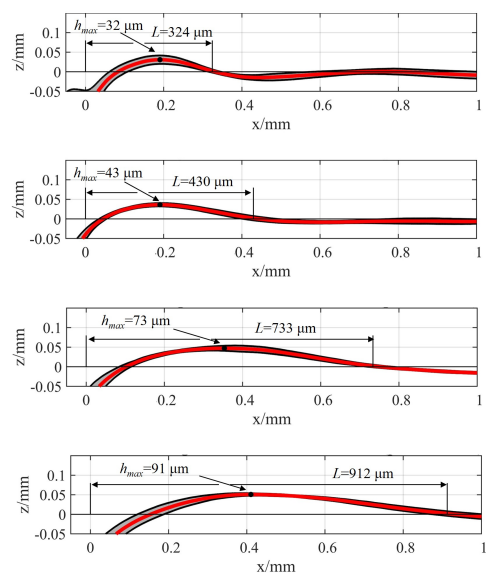

Figure 5: Edge effect morphology as a function of the normalized enthalpy of the remelting step, (top) view on HS, (bottom) 2.5D height map based on tactile measurements. Variable parameters: (a) $P=100 \mathrm{~W}, v=1100 \mathrm{~mm} / \mathrm{s}$, normalized enthalpy $\Delta H / h_{s}=2.2$, (b) $P=100 \mathrm{~W}, v=700 \mathrm{~mm} / \mathrm{s}$, $\Delta H / h_{s}=2.7$, (c) $P=150 \mathrm{~W}, v=700 \mathrm{~mm} / \mathrm{s}, \Delta H / h_{s}=4.1$, (d) $P=175 \mathrm{~W}, v=600 \mathrm{~mm} / \mathrm{s}, \Delta H / h_{s}=5.2$. Fixed parameters: $\alpha=5^{\circ}, N_{R}=4, h=35 \mu \mathrm{m}$. 


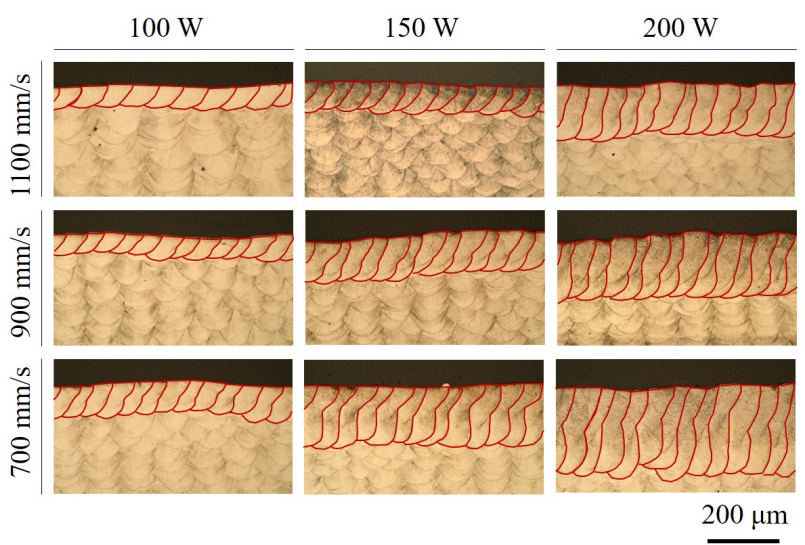

Figure 6: Melt pool morphology as a function of process parameters variation, MP after remelting marked in red $\left(\alpha=5^{\circ}, N_{R}=4, h=35 \mu \mathrm{m}\right)$.

be expected. Moreover, during scanning material is relocalised from the zone of scan vector start towards the zone of its end. A larger volume of melt pool would then also promote the occurrence of elevated edges. Furthermore, due to the denudation effect described in detail by Matthews et al. (2016), more powder can be drawn towards the melt pool, increasing the edge size.

Sample corners are emphasized witnesses of the edge effect, as they present the worst case scenario. The corners shown in Fig. 5 are generated after scanning under $45^{\circ}$, leading to a severe heat accumulation in this area of scan vectors ends. Hence scanning parallel or perpendicular to the edges might improve the geometrical precision of the corners. Moreover, $P$ and $v$ variation leads to a different melt pool width compared to the bulk material. The offset of the contours from the CAD model should be thus adapted accordingly.

\subsubsection{Effect on the microstructure}

The effect of the laser spot diameter variation on the melt pool (MP) behavior seems to be negligible for the $5^{\circ}$ samples, as the height difference from the focal plane remains within $650 \mu \mathrm{m}$. Most of the samples show a constant melt pool depth $\left(d_{M P}\right)$, but in some samples a slight $d_{M P}$ variation was observed, increasing between the beginning and end of the scanning. This follows a linear trend, which would suggest an effect of heat accumulation throughout the scanning. The maximal observed variation $\Delta d_{M P}$ was $2.2 \mu \mathrm{m}$ per millimeter of remelted surface, which is negligible compared to the standard deviation of $d_{M P}$ (typically about 5-10 $\mu \mathrm{m}$, depending on the used parameters).

The effect of the $P$ and $v$ variation on the MP morphology, on the other hand, is significant. Fig. 6 presents MP after remelting, ranging from a conduction melting mode $\left(d_{M P}=46.6 \mu \mathrm{m} \pm 2.3 \mu \mathrm{m}\right)$ up to a deep keyhole mode $\left(d_{M P}=222.0 \mu \mathrm{m} \pm 18.0 \mu \mathrm{m}\right)$. However, despite the significant depth, MP seem to be very stable. Only in the two samples with the highest energy input $(P=200 \mathrm{~W}, v=700 \mathrm{~mm} / \mathrm{s}$ and $P=175 \mathrm{~W}, v=600 \mathrm{~mm} / \mathrm{s})$ a low amount of subsurface defects (keyhole porosity) and rare micro-cracks $(<50 \mu \mathrm{m})$ were observed. Note that the metallographic cross-sections in Fig. 6 are in a plane oriented $45^{\circ}$ to the scanning direction, so they appear wider by a factor $1 / \sin \left(45^{\circ}\right)=\sqrt{2} \approx 1.4$.

\subsubsection{Effect on the microhardness}

The reference microhardness of the bulk material $H_{\text {ref }}$ was evaluated as $383 \mathrm{HV} \pm 6 \mathrm{HV}$. The value is based on 30 measurements in the middle zone of the samples, hence in the as-built state. Examples of typical profiles of subsurface microhardness variations after remelting are given in Fig. 7a-c, with $d_{M P}$ illustrated in orange. In the close vicinity of the surface, a slight decrease of $5-10 \%$ was observed, followed by an increase up to $+26 \%$ in some samples. A variation was considered significant when $H$ was outside the range of three standard deviations $(s)$ from $H_{r e f}$ (Eq. 3). Assuming a Gaussian distribution, $99.7 \%$ of $H_{\text {ref }}$ should be situated in this range.

$$
\left|H-H_{r e f}\right|>3 \cdot s
$$




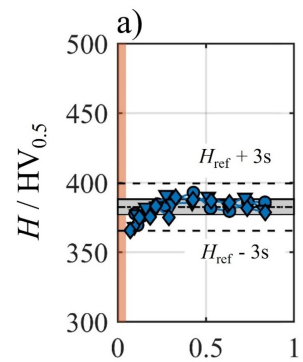

b)

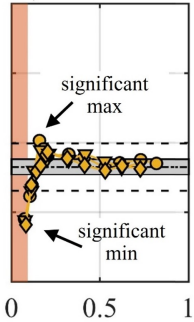

c)

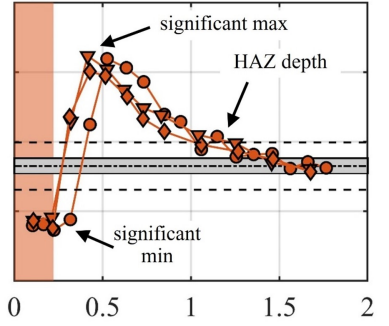

Distance from the surface / $\mathrm{mm}$

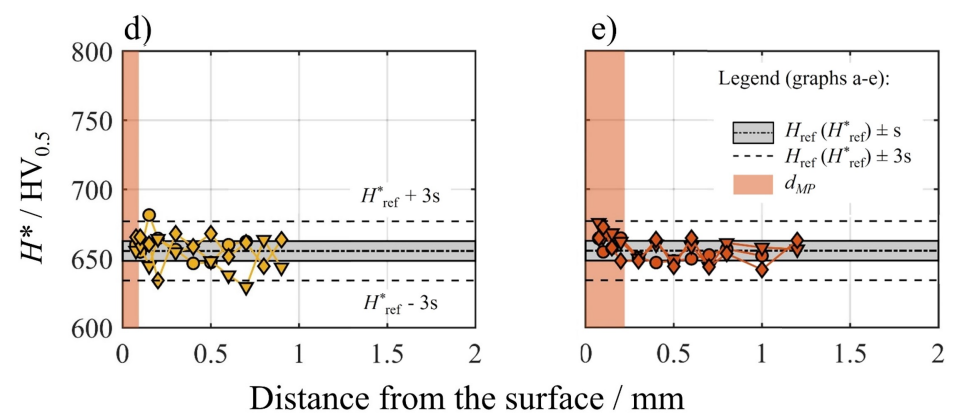

Figure 7: Microhardness variation after remelting, with typical microhardness profiles for a sample in a) conduction melting mode $(P=100 \mathrm{~W}$, $v=1100 \mathrm{~mm} / \mathrm{s}), \mathrm{b})$ transient mode at the threshold between conduction and keyhole $(P=100 \mathrm{~W}, \nu=700 \mathrm{~mm} / \mathrm{s}), \mathrm{c}) \mathrm{keyhole}$ mode $(P=175 \mathrm{~W}$, $v=600 \mathrm{~mm} / \mathrm{s})$ and $(\mathrm{d}, \mathrm{e})$ corresponding to $(\mathrm{b}, \mathrm{c})$ after heat treatment.
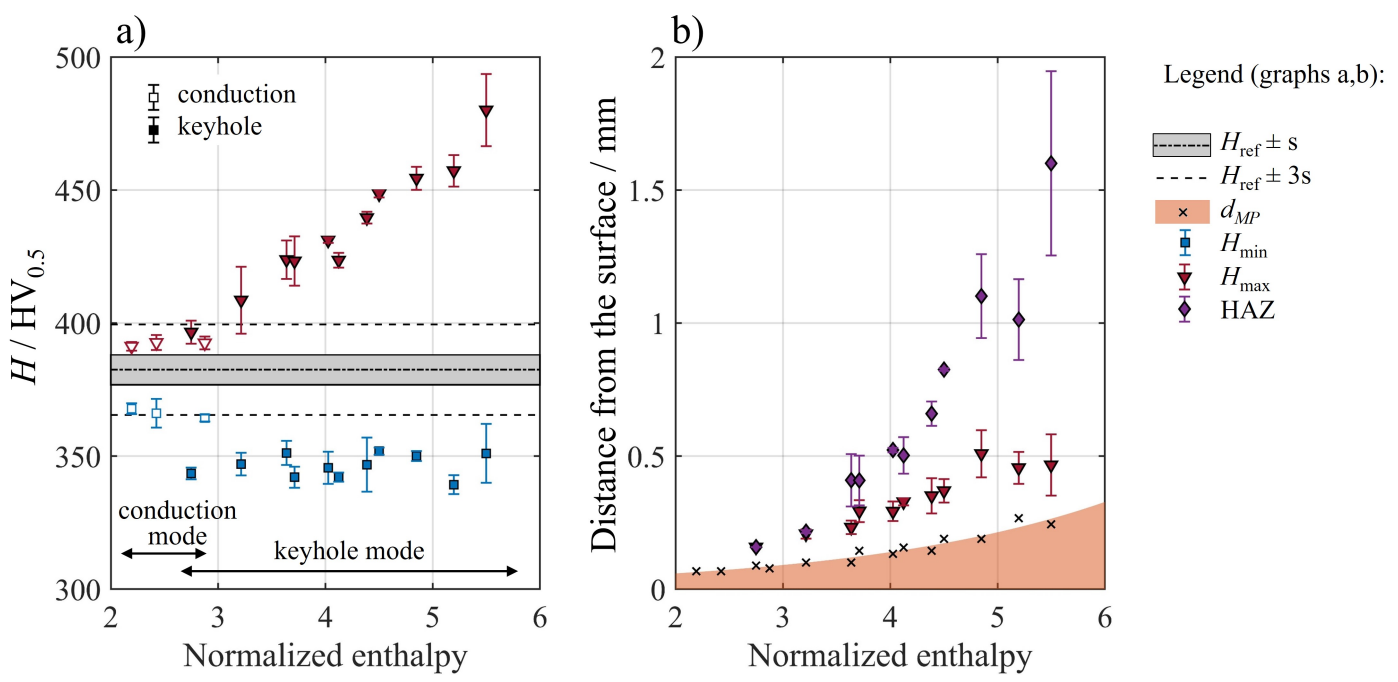

Figure 8: Microhardness variation depending on the heat input from remelting, with: (a) minimal $\left(H_{\min }\right)$ and maximal $\left(H_{\max }\right)$ detected microhardness as a function of enthalpy, (b) location of the significant $H_{\max }$ and HAZ with respect to the fitted MP boundaries in orange. 
The MP depth and maximal $H$ variation as a function of heat input are shown in Fig. 8. Samples in conduction melting mode (e.g. Fig. 7a) were typically within $\pm 5 \%$ variation from the reference. Fig. 8a shows an average $H$ decrease of conduction MP of $4.3 \%$ and very little heat affected zone (HAZ) with a mean increase by $2.5 \%$. $H$ returned to the range of $H_{\text {ref }}$ just below $d_{M P}$ (Fig. 7a).

The observed minimal microhardness $H_{\min }$ in the samples in the keyhole mode dropped in average by $9.4 \%$ compared to $H_{r e f}$. It remained constant during the whole $d_{M P}$ (Fig. 7c) and did not vary significantly even with an increasing heat input (Fig. 8a). The difference in $H_{\text {min }}$ between conduction and keyhole mode can be explained by the measurement location of the first indentation $(\approx 50-75 \mu \mathrm{m})$ below the surface, which is comparable to $d_{M P}$ of some samples with the lowest heat input. In some cases, the indentation was thus made at the MP edge. The real $H_{\min }$ could be hence expected to be similar for both melting modes and with only a little variation throughout the MP.

A significant increase in $H$ was observed only in keyhole mode (Fig. 8a). As shown in Figs. 7c,8b, the location of the maximal observed microhardness $\left(H_{\max }\right)$ was systematically below the MP boundary (indicated in orange). The distance from the surface as well as the value of $H_{\max }$ increases with increasing heat input, up to $+26 \%$ $\left(\max \left(H_{\max }\right)=480 \mathrm{HV} \pm 13 \mathrm{HV}\right)$. As illustrated in Fig. $7 \mathrm{c}$, the depth of the heat affected zone $(\mathrm{HAZ})$ is defined as the deepest significantly increased $H$ (see Eq. 3). The depth of HAZ increases with heat input, however with a steeper slope than $d_{M P}$ (Fig. 8b).

The increase in $H$ is probably due to the high melting temperatures occurring in the keyhole regime. An SEM analysis revealed that the microstructural cell size is comparable in the zone of increased $H$ and in the reference bulk zone (several millimeters below the top surface). However, a slight increase of molybdenum was observed in the zone of increased $H$. This might indicate a formation of precipitates during the high remelting temperatures. This $H$ variation accompanied by a $H$ gradient might not be suitable for some high-end applications. Remelting in a conduction mode, or just at the threshold between the two modes, is thus more suitable. On the other hand, in order to meet industrial requirements, critical parts are typically heat treated after manufacturing. A heat treatment as it was suggested by Elangeswaran et al. (2020) was applied to selected samples. In accordance with the results reported by Elangeswaran et al., the reference bulk hardness after heat treatment $H_{r e f}^{*}$ increased to $656 \mathrm{HV} \pm 7 \mathrm{HV}$. Fig. $7 \mathrm{~d}$,e shows that the $H$ variations were reduced within three standard deviation from $H_{r e f}^{*}$.

\subsection{Laser remelting of other sample geometries}

Based on the previous sections, the parameters for the remelting step were selected as follows: $P=100 \mathrm{~W}$, $\nu=700 \mathrm{~mm} / \mathrm{s}, h=35 \mu \mathrm{m}$ and $N_{R}=4$. They lead to an optimal combination of a low surface texture $\left(S_{a}=6.2 \mu \mathrm{m}\right)$ and a very stable melt pool $\left(d_{M P}=91.5 \mu \mathrm{m} \pm 3.9 \mu \mathrm{m}\right)$ at the transition between conduction and keyhole regime. Moreover the impact on the microstructure is also low: only a slight $H$ increase of $5 \%$ compared to $H_{r e f}$ was observed. Finally the edge morphology is also in the lower range of the observed samples: $h_{\text {max }}=36.9 \mu \mathrm{m} \pm 4.1 \mu \mathrm{m}$ and $L=430 \mu \mathrm{m}$.

After selecting the optimal process parameters, the transferability to other sample geometries was investigated through (1) variation of the width of the $5^{\circ}$ block (Fig. 2a) and (2) application to the sinusoidal sample geometry (Fig. 2b), representing a great challenge.

\subsubsection{Variation of sample width}

Only a slight impact on the surface quality was observed between the sample width $w=6 \mathrm{~mm}$ and $30 \mathrm{~mm}$, this was likely within the process reproducibility boundaries. For $N_{R}=2,5 \%$ increase was observed (from $S_{a}=6.61 \mu \mathrm{m}$ to $6.95 \mu \mathrm{m}$ ) and $19 \%$ increase for $N_{R}=4$ (from $S_{a}=4.90 \mu \mathrm{m}$ to $5.81 \mu \mathrm{m}$ ). Also $H$ and $d_{M P}$ varied only to a lesser extent. Therefore this parameter set was found to be relatively stable in the vicinity of the focal plane $\left(\alpha=5^{\circ}\right.$, defocus $|\Delta f|<0.65 \mathrm{~mm})$, but a higher degree of defocusing should be investigated as well. This is realized using the sinusoidal sample, involving a $4 \mathrm{~mm}$ height step $(|\Delta f|<2 \mathrm{~mm})$.

\subsubsection{Sinusoidal sample}

The texture of the horizontal surfaces in the as-built (AB) state is affected mainly by the spatter particles, waviness along the scan tracks and between the scan tracks (Fig. 10c,d). The inclined AB surface suffers from a large amount of attached powder particles, combined with the staircase effect and possible elevated edges at the border of each scanned area.

Figs. 9 and 10a,b show that manual powder brushing and subsequent surface remelting (samples $\mathrm{MB}+\mathrm{R}$ ) can significantly improve the surface texture of sinusoidal samples, despite a high level of defocusing. As given in Fig. 9, 


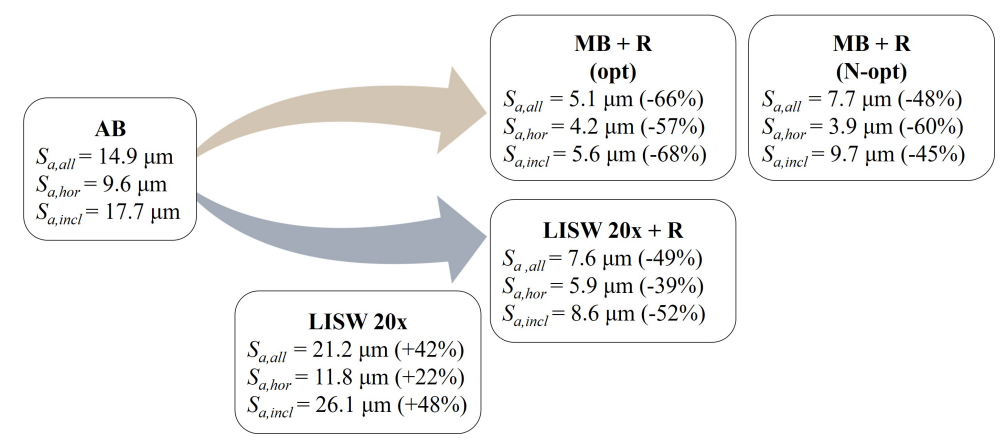

Figure 9: Flowchart of the processes and arithmetical mean height of the overall surface $S_{a, a l l}$, inclined $S_{a, \text { incl }}$ and both horizontal surfaces $S_{a, h o r}$ of the sinusoidal samples. The relative values are with respect to AB. AB: as-built state, LISW: processing with laser-induced shock waves, MB: manual brushing, R: remelting, opt: optimal parameter set $(100 \mathrm{~W}, 700 \mathrm{~mm} / \mathrm{s}), \mathrm{N}$-opt: non-optimal parameter set $(150 \mathrm{~W}, 1100 \mathrm{~mm} / \mathrm{s})$.

after remelting with the optimal parameters $(P=100 \mathrm{~W}, v=700 \mathrm{~mm} / \mathrm{s})$, the arithmetical mean deviation of the inclined surface $\left(S_{a, i n c l}\right)$ could be decreased by $68 \%$ with respect to the AB state, from $17.7 \mu \mathrm{m}$ to $5.6 \mu \mathrm{m}$. As a consequence, the texture of inclined and horizontal surfaces after remelting become comparable $\left(S_{a \text {,incl }}=5.6 \mu \mathrm{m}\right.$ and $\left.S_{a, \text { hor }}=4.2 \mu \mathrm{m}\right)$. It should be noted that the part-to-part process variability of such remelted surfaces is typically up to $S_{a}=1 \mu \mathrm{m}$. Due to the large amount of investigated parameters and the time and cost expensive nature of the hybrid LPBF processing, the samples were not produced multiple times in this study.

Besides the optimal parameter set, a second one was also applied on the sinusoidal sample remelting, identical to the sample building parameters $(P=150 \mathrm{~W}, v=1100 \mathrm{~mm} / \mathrm{s})$. As shown in Fig. 9 , the surface texture appears to be almost identical on the horizontal surfaces after remelting with either parameter set. Nevertheless, undesired surface texturing was observed for the non-optimal parameter set around the maximal surface slope (Fig. 10a,b). The texture direction corresponds to an intermediate direction between the scanning direction and surface gradient. This is probably due to the combination of deeper MP and a high inclination angle $\left(\alpha_{\max }=40^{\circ}\right)$, making the MP flow down due to gravity. Moreover, the high scanning speed can further increase the MP instabilities and oscillations. Another explanation could be high reflections due to the high slope angle. However, this hypothesis was excluded after building samples at different positions on the base plate, which did not affect the surface texturing. Similar observations were also made for inclined plane surfaces with $\alpha \geq 30^{\circ}$, which were remelted with the mentioned non-optimal parameter set.

\subsection{Hybrid processing of sinusoidal samples}

In order to study the effect of the hybrid processing on the surface integrity, two ways of powder removal were applied to the sinusoidal samples: removal by manual brushing (MB) and by laser-induced shock waves (LISW). The first one is time-consuming, as it requires the LPBF process interruption, machine door opening, manual powder removal and re-running of the inert gas cycle. For an industrial application, the in-situ integration of all three steps (LPBF, powder removal by LISW, surface remelting) is a more suitable solution.

Figs. 10 and 11 illustrate the surface and subsurface quality of the sinusoidal samples in the four observed states: as-built ( $\mathrm{AB}$ ), after powder removal using laser-induced shock waves (LISW), and after remelting preceded either by powder removal using LISW or MB.

\subsubsection{Powder removal using LISW}

As reported in a previous work Metelkova and Ordnung et al. (2021), insufficient powder removal can cause significant quality issues. The metal powder remaining on the inclined surface would be melted during the remelting step. Moreover, it would be attached to the laser polished surface, forming undesired freeform structures at the edge of the remelted zone.

The sinusoidal sample with $4 \mathrm{~mm}$ height step and a maximal slope angle $\alpha_{\max }=40^{\circ}$, could be cleaned entirely after 10 and more LISW powder removal passes. After five passes, the surface is almost powder-free, except for a small amount in one corner of BS. Figs. 10 and 11 show samples after 20 LISW passes. 

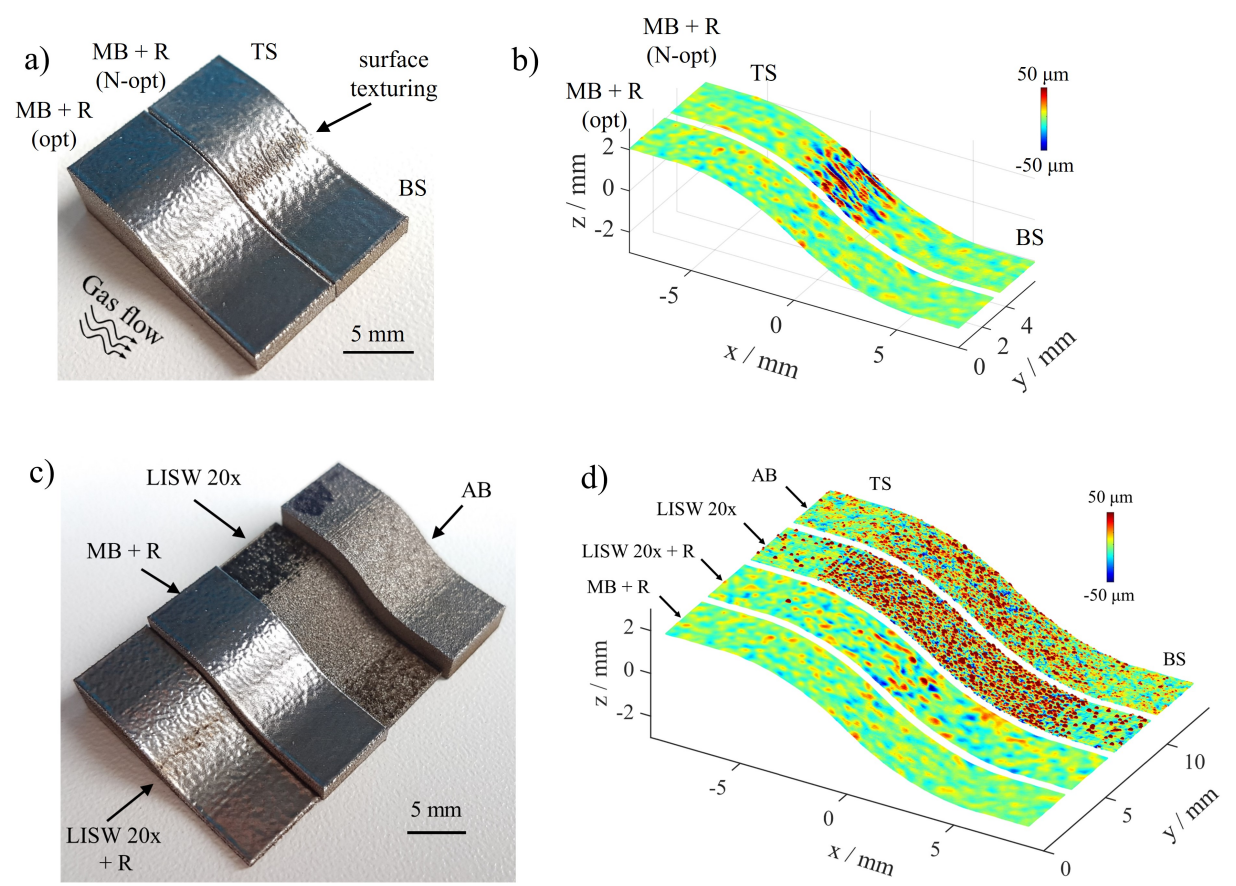

Figure 10: Photos $(\mathrm{a}, \mathrm{c})$ and height maps $(\mathrm{b}, \mathrm{d})$ based on 2.5D tactile data of the sinusoidal samples. AB: as-built state, LISW: processing with laser-induced shock waves, MB: manual brushing, R: remelting, opt: optimal parameter set $(100 \mathrm{~W}, 700 \mathrm{~mm} / \mathrm{s})$, N-opt: non-optimal parameter set $(150 \mathrm{~W}, 1100 \mathrm{~mm} / \mathrm{s})$.

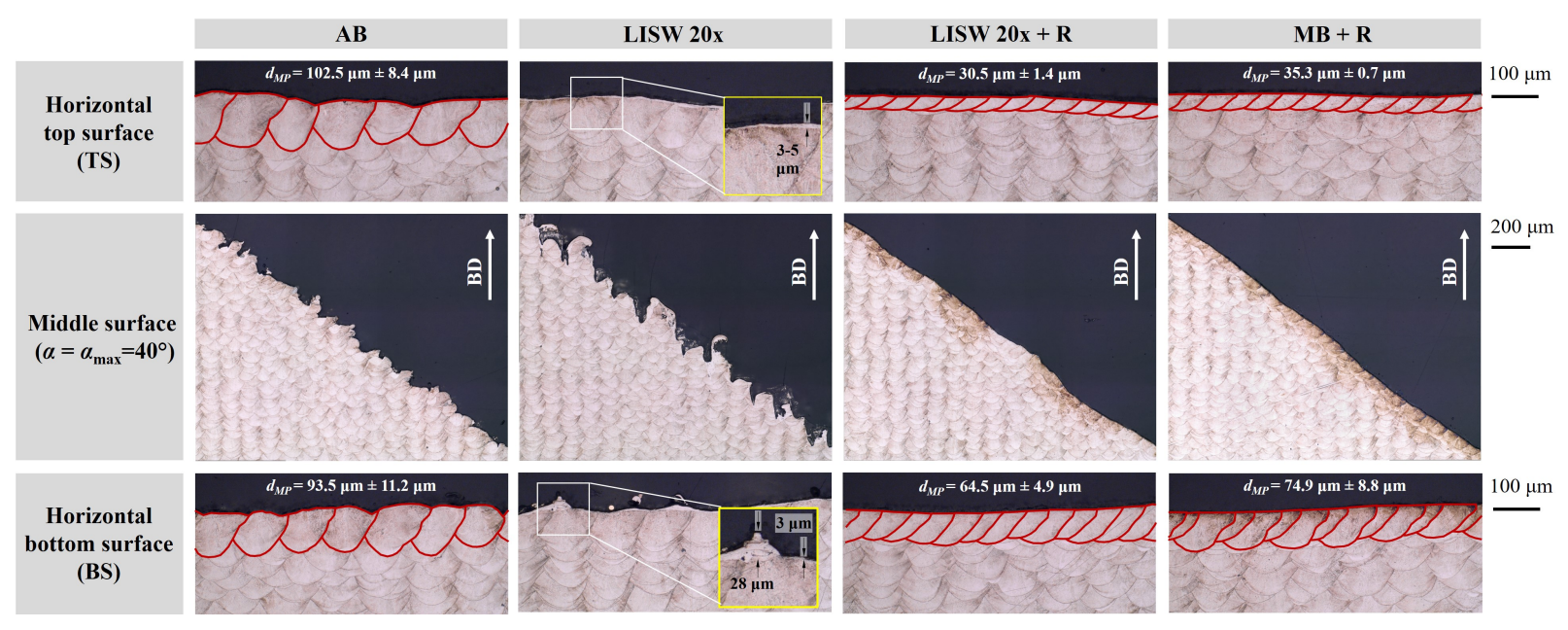

Figure 11: Microstructure of the sinusoidal samples. AB: as-built state, LISW: processing with laser-induced shock waves, MB: manual brushing, R: remelting, BD: building direction, $d_{M P}$ : melt pool depth. 
During the powder removal step, the surface texture increases with an increasing number of LISW passes, on both horizontal and inclined surfaces (compare "AB" and "LISW 20x" in Fig. 11). As illustrated in Fig. 9, $S_{a \text {,all }}$ calculated over the full measured surface, increased from $14.9 \mu \mathrm{m}$ in the AB state till $21.2 \mu \mathrm{m}$ after $20 \mathrm{LISW}$ passes. The increase is stronger on the inclined surfaces $(+48 \%)$ compared to the horizontal surfaces $(+22 \%)$.

As shown in Fig. 11 ("LISW 20x"), ablation of the horizontal surfaces (TS, BS) results in a quite continuous and regular recast layer, about 3-5 $\mu \mathrm{m}$ thick. Occasionally, new surface asperities were observed. These are probably related to instabilities during the ablation process and melt expulsion under the strong recoil pressure of LISW. Most of the newly created asperities were observed on the inclined surface, often close to the "stair" edge from the sample discretization into layers. As a matter of fact, on rough surfaces significantly more instabilities occur, leading to deepening of existing surface irregularities and more material expulsion.

\subsubsection{Laser remelting after LISW powder removal}

Figs. 9-11 show that despite a rough surface texture after LISW, the remelting step was able to smoothen the surface from $S_{a, a l l}=14.9 \mu \mathrm{m}$ to $7.6 \mu \mathrm{m}$, corresponding to about $49 \%$ reduction compared to the AB state. However, a certain degree of residual waviness was observed after remelting of LISW surfaces ("LISW 20x + R" in Fig. 10d), compared to $\mathrm{MB}+\mathrm{R}$ surfaces. This waviness is found mostly on the inclined surface, especially in the vicinity of $\alpha_{\max }$ (Fig. 10d). Consequently, the hybrid processing leads to a slightly higher texture compared to the manual powder removal followed by remelting $\left(S_{a, \text { all }}=5.1 \mu \mathrm{m}\right)$.

Different observations were made by Metelkova and Ordnung et al. (2021), for samples with $\alpha=5^{\circ}$, made from the same material, in the same machine and process parameters. The authors did not observe a significant impact of the number of LISW powder removal passes on the surface quality after remelting with identical process parameters to this study. The main difference was the defocusing distance, as remelting of a $5^{\circ}$ surface with $d=1.3 \mathrm{~mm}$ corresponds to a defocus $|\Delta f|<0.65 \mathrm{~mm}$, compared to $|\Delta f|<2 \mathrm{~mm}$ for the sinusoidal sample $(d=4 \mathrm{~mm})$. The remelting step is thus more efficient when scanning closer to the focal plane.

The microstructure does not seem to be significantly impacted by the powder removal step using LISW (Fig. 11). The thin recast layer $(3-5 \mu \mathrm{m})$ after PW processing is entirely remelted by the following remelting step $\left(d_{M P}=30\right.$ $75 \mu \mathrm{m}$ ). In case of both powder removal strategies (by MB or LISW), deeper melt pools were observed after remelting of BS compared to TS (see Fig. 11). This observation does not correspond to the findings of Metelkova et al. (2018), investigating the defocusing sign. Negative defocusing sign refers to the focal plane situated inside the material and positive sign refers to the focal plane above the material. The authors found that negative defocus (here interaction with TS) would lead to deeper melt pools compared to positive defocus (interaction with BS). However, in the present study an additional factor has to be taken into account: the surface position with respect to the gas flow. The gas flow stream arrives directly to TS but BS is partially shielded, as BS is situated in the lee of the inclined surface. This could cause a lower cooling efficiency by the gas flow, which would lead to more overheating. This hypothesis is further supported by the a larger number of instabilities and melt expulsion spatters after PW laser processing of BS, which might be interpreted as signs of a higher energy input.

Although remelting after MB leads to a slightly better surface texture compared to remelting after LISW, the additional processing time is significantly higher. MB requires releasing the inert atmosphere (about 5 min), manual brushing of the powder (about $2 \mathrm{~min}$ ) and re-running the inert cycle (about $30 \mathrm{~min}$ ). On the other hand, in case of the samples from this study, powder removal by LISW takes about $5.6 \mathrm{~s}$ per pass plus about $10 \mathrm{~s}$ to switch between the two lasers. Hence, 20 powder removal passes take only $122 \mathrm{~s}$, making it about 18x more efficient than MB. Even though the exact processing time depends on the component size and geometry, using LISW will remain significantly faster. Moreover, contrary to MB, only a limited assistance of the operator is required for LISW, which could be fully automated if needed.

As such, improving the surface roughness of LPBF parts by LISW powder removal and subsequent laser remelting will be faster and cheaper than doing the same by manual brushing (MB) and laser remelting. It will also be faster and cheaper than applying other post-LPBF finishing methods like e.g. post-process machining (CNC milling, grinding or abrasive flow machining). Moreover, LPBF components have often complex freeform shapes, which would otherwise require manual grinding and polishing. This dual-laser setup can be implemented to other LPBF machines as well. This solution to improve surface roughness and geometrical accuracy of components is thus complementary to recommendations of Chahal and Taylor (2020) regarding a standard LPBF process. 
An additional point to be taken into account concerns the build cylinder offset $\Delta Z$, as it directly affects the end positioning of the laser spot size. The XY calibration is performed for the in-focus height. Working outside of the laser focus plane is therefore affected by the incident angle of the laser beam and the distance from the centre of the scanning field. For instance, considering the optical system used in this study, on a $90 \mathrm{~mm}$ x $90 \mathrm{~mm}$ base plate, a compensation up to $55 \mu \mathrm{m}$ per millimetre of $\Delta Z$ has to be made. These modifications can be implemented directly in the CAD model. Another possible solution would be to use an optical system with a dynamic focusing unit enabling 3D scanning and focus shift during scanning.

\section{Conclusions}

This paper presents an investigation of the surface integrity of samples produced by laser powder bed fusion (LPBF), which were subjected to in-situ powder removal using laser-induced shock waves (LISW) and subsequent surface remelting. This study demonstrates that the described method is a fast and efficient solution for quality improvement of inclined up-facing surfaces, applicable to various sample geometries.

The first part of the study deals with surface remelting alone, as the powder was removed from inclined surfaces by manual brushing without altering the sample's surface quality. The remelting was carried out using a continuous wave laser. The investigation focused on the effect of process parameters on the surface texture, geometrical accuracy, melt pool morphology and microhardness alterations. The effect of scanning speed on the surface texture was found to be significant. The geometrical accuracy of the edges and corners was found to be strongly influenced by the heat input. It led to a slight increase of the maximal edge height, and a significant increase of the edge length. Remelting with melt pools in the conduction mode did not led to significant microhardness alterations. On the other hand, deep keyhole melt pools showed a constant drop in microhardness $(9.4 \%)$ with respect to the bulk microhardness. Below the melt pool depth, an increase of microhardness up to $+26 \%$ was noted. These microhardness variations were removed after a solutionizing and age hardening heat treatment.

After the remelting study, the "hybrid" dual-laser processing method was investigated, using pulsed laser to remove powder by LISW. Sinusoidal samples with a $4 \mathrm{~mm}$ height step and slope angle up to $40^{\circ}$ were successfully processed. Its overall surface roughness $S_{a \text {,all }}$ could be reduced by $42 \%$, from $14.9 \mu \mathrm{m}$ in the as-built state (after LPBF) to $7.6 \mu \mathrm{m}$ after remelting. The improvement was observed to be higher on inclined surfaces (52\%) compared to horizontal surfaces (39\%). Nevertheless, the LISW powder removal process has to be fully optimized prior to the remelting step. Otherwise, substantial amount of powder particles can remain attached to the laser polished surface and thus compromise the final quality.

The subsurface integrity of the remelted samples did not show any major defects related to the LISW processing. The depth affected by this pulsed laser processing is significantly lower compared to the melt pool depth during remelting. Any defect would hence be eliminated by this subsequent step.

Roughness reduction is an important factor affecting the component performance, especially in case of fatigue loading. This study is a part of ongoing research. Currently, a preliminary investigation on fatigue loading of remelted samples from various materials is carried out, showing very promising results. In the framework of future research, special focus will be given to up-scaling of this method in order to enable its application to industrial components.

\section{Acknowledgements}

This research was funded by the H2020-MSCA-ITN-2016 project PAM ${ }^{2}$ (Precision Additive Metal Manufacturing), EU Framework Programme for Research and Innovation H2020 [Grant $n^{\circ} 721383$ ], and by the Research Foundation Flanders (FWO) [Grant n ${ }^{\circ}$ S009319N] (FWO-SBO project "HIPASS"). Moreover, the authors would like to acknowledge the help of Pushkar Dhekne with the Scanning Electron Microscopy measurements.

Ben-Artzy, A., Reichardt, A., Borgonia, J.-P., Dillon, R.P., McEnerney, B., Shapiro, A.A. and P. Hosemann. 2021. "Compositionally graded SS316 to C300 Maraging steel using additive manufacturing." Mater. Des. 201: 109500. Bhaduri, D., Penchev, P., Batal, A., Dimov, S., Soo, S.L., Sten, S., Harrysson, U., Zhang, Z., and Dong, H. 2017. "Laser polishing of 3D printed mesoscale components." Appl. Surf. Sci. 405:29-46.

Boban, J., and A. Ahmed. 2021. "Improving the surface integrity and mechanical properties of additive manufactured stainless steel components by wire electrical discharge polishing." J. Mater. Process. Technol. 291:117013. 
Boschetto, A., Bottini, L. and F. Veniali. 2017. "Roughness modeling of AlSi10Mg parts fabricated by selective laser melting." J. Mater. Process. Technol. 241:154-163.

Campbell, R.I., Martorelli, M. and H.S. Lee. 2002. "Surface roughness visualisation for rapid prototyping models." Comput. Aided Des. 34:717-725.

Chahal, V. and R.M. Taylor. 2020. "A review of geometric sensitivities in laser metal 3D printing." Virtual Phys. Prototyp. 15(2): 227-241.

Colosimo, B.M., Grossi, E., Caltanissetta, F. and M. Grasso. 2020. ”Penelope: A Novel Prototype for In Situ Defect Removal in LPBF." JOM 72:1332-1339.

Du, W., Bai, Q. and B. Zhang. 2018. "Machining characteristics of $18 \mathrm{Ni}-300$ steel in additive/subtractive hybrid manufacturing." Int. J. Adv. Manuf. Techn. 95:2509-2519.

Elangeswaran, C., Gurung, K., Koch, R., Cutolo, A. and B. Van Hooreweder. 2020. "Post-treatment selection for tailored fatigue performance of $18 \mathrm{Ni} 300$ maraging steel manufactured by laser powder bed fusion." Fatigue Fract. Eng. Mater. Struct. 43:2359-2375.

Ghorbani, J., Li, J., and A.K. Srivastava. 2020. ”Application of optimized laser surface re-melting process on selective laser melted 316L stainless steel inclined parts.” J. Manuf. Process. 56:726-734.

Gora, W.S., Tian, Y., Cabo, A.P., Ardron, M., Maier, R.R., Prangnell, P., Weston, N.J., and Hand, D.P. 2016. "Enhancing surface finish of additively manufactured Titanium and cobalt chrome elements using laser based finishing." Phys. Procedia 83:258-263.

International Organization for Standardization. 2016. "ISO 16610-31: Geometrical product specifications (GPS) Filtration - Part 31: Robust profile filters: Gaussian regression filters.” Geneva, Switzerland.

International Organization for Standardization. 2015. "ISO 17296-2: Additive manufacturing - General principles Part 2: Overview of process categories and feedstock." Geneva, Switzerland.

International Organization for Standardization. 2012. "25178-2: Geometrical Product Specification (GPS) - Surface Texture: Areal - Part 2: Terms, Definitions and Surface Texture Parameters.” Geneva, Switzerland.

King, W.E., Barth, H.D., Castillo, V.M., Gallegos, G.F., Gibbs, J.W., Hahn, D.E., Kamath, C. and A.M. Rubenchik. 2014. "Observation of keyhole-mode laser melting in laser powder-bed fusion additive manufacturing." J. Mater. Process. Technol. 214:2915-2925.

Kuisat, F., Lasagni, F., and A.F. Lasagni. 2021. "Smoothing additive manufactured parts using ns-pulsed laser radiation." Prog. Addit. Manuf.

Kumstel, J., Kirsch, B. 2013. "Polishing titanium- and nickel-based alloys using cw-laser radiation." Phys. Proc. 41:362-371.

Lamikiz, A., Sanchez, J.A., Lopez de Lacalle, L.N. and J.L. Arana. 2007. "Laser polishing of parts built up by selective laser sintering." Int. J. Mach. Tools Manuf. 47:2040-2050.

Leach, R.K., Bourell, D., Carmignato, S., Donmez, A., Senin, N. and W. Dewulf. 2019. "Geometrical metrology for metal additive manufacturing." CIRP Ann. 68:677-700.

Lee, J.Y., Nagalingam, A.P., and S. H. Yeo. 2021. "A review on the state-of-the-art of surface finishing processes and related ISO/ASTM standards for metal additive manufactured components." Virtual Phys. Prototyp. 16:68-96.

Ma, C.P., Guan, Y.C., and Zhou, W. 2017. "Laser polishing of additive manufactured Ti alloys." Opt. Lasers Eng. 93:171-177.

Marimuthu, S., Triantaphyllou, A., Antar, M., Wimpenny, D., Morton, H., and M. Beard. 2015. 'Laser polishing of selective laser melted components.” Int. J. Mach. Tools Manuf. 95:97-104.

Matthews, M.J., Guss, G., Khairallah, S.A., Rubenchik, A.M., Depond, P.J., and W.E. King. 2016. "Denudation of metal powder layers in laser powder bed fusion processes." Acta Mater. 114:33-42.

Metelkova, J., de Formanoir, C., Haitjema, H., Witvrouw, A., Pfleging, W. and B. Van Hooreweder. 2019. "Elevated edges of metal parts produced by laser powder bed fusion: characterization and post-process correction." Proc. aspe-Euspen.

Metelkova, J., Kinds, Y., Kempen, K., de Formanoir, C., Witvrouw, A. and B. Van Hooreweder. 2018. ”On the influence of laser defocusing in Selective Laser Melting of 316L.” Addit. Manuf. 23:161-169.

Metelkova, J., Ordnung, D., Kinds, Y., Witvrouw, A. and B. Van Hooreweder. 2020. "Improving the quality of upfacing inclined surfaces in laser powder bed fusion of metals using a dual laser setup." Procedia CIRP 94:266-269.

Metelkova, J. and Ordnung, D., Kinds, Y. and B. Van Hooreweder. 2021. "Novel strategy for quality improvement of up-facing inclined surfaces of LPBF parts by combining laser-induced shock waves and in-situ laser remelting." $J$. 
Mater. Process. Technol. 290:116981.

Newton, L., Senin, N., Chatzivagiannis, E., Smith, B., and R. Leach. 2020. "Feature-based characterisation of Ti6Al4V electron beam powder bed fusion surfaces fabricated at different surface orientations." Addit. Manuf. 35:101273.

Ramos-Grez, J.A., and Bourell, D.L. 2004. "Reducing surface roughness of metallic freeformfabricated parts using non-tactile finishing methods." Int. J. Mat. Prod. Technol. 21.

Schmidt, M., Merklein, M., Bourell, D., Dimitrov, D., Wegener, T.H.K., Overmeyer, L., Vollertsen, F., and G. Levy. 2017. "Laser based additive manufacturing in industry and academia." CIRP Annals 66:561-583.

Sing, S. L., and W.Y. Yeong. 2020. "Laser powder bed fusion for metal additive manufacturing: perspectives on recent developments." Virtual Phys. Prototyp. 15:359-370.

Sinico, M., Witvrouw, A. and W. Dewulf. 2019. "Influence of the particle size distribution on surface quality of Maraging 300 parts produced by Laser Powder Bed Fusion.” Proc. aspe-Euspen.

Strano, G., Hao, L., Everson, R.M., K.E. Evans. 2013. "Surface roughness analysis, modelling and prediction in selective laser melting." J. Mater. Process. Technol. 213:589-597.

Webster, S., Lin, H., Carter III, F.M., Ehmann, K., and Cao, J. 2021. "Physical mechanisms in hybrid additive manufacturing: A process design framework." J. Mater. Process. Technol. 291:117048.

Wüst, P., Edelmann, A., and R. Hellmann. 2020. "Areal surface roughness optimization of maraging steel parts produced by hybrid additive manufacturing." Materials 13:418.

Yasa, E., Kruth, J.-P., and J. Deckers. 2011. "Manufacturing by combining Selective Laser Melting and Selective Laser Erosion/laser re-melting." CIRP Annals 60:263-266.

Yasa, E., and J.P. Kruth. 2011. "Application of laser re-melting on selective laser melting parts." Adv. Prod. Eng. 6:259-270.

Yung, K.C., Xiao, T.Y., Choy, H.S., Wang, W.J., and Cai, Z.X. 2018. ”Laser polishing of additive manufactured CoCr alloy components with complex surface geometry.” J. Mater. Process. Technol. 262:53-64.

Yung, K.C., Zhang, S.S., Duan, L., Choy, H.S., Cai, Z.X. 2019. 'Laser polishing of additive manufactured tool steel components using pulsed or continuous-wave lasers." Int. J. Adv. Manuf. Technol. 105:425-440. 